\title{
Chaos Control and Anticontrol of the Output Duopoly Competing Evolution Model
}

\author{
Zhaoqing Li, ${ }^{1}$ Yongping Zhang, ${ }^{1}$ and Tongqian Zhang ${ }^{2}$ \\ ${ }^{1}$ School of Mathematics and Statistics, Shandong University, Weihai 264209, China \\ ${ }^{2}$ College of Mathematics and Systems Science, Shandong University of Science and Technology, Qingdao 266590, China \\ Correspondence should be addressed to Yongping Zhang; ypzhang@sdu.edu.cn
}

Received 4 July 2017; Revised 12 September 2017; Accepted 10 October 2017; Published 6 November 2017

Academic Editor: Rigoberto Medina

Copyright (c) 2017 Zhaoqing Li et al. This is an open access article distributed under the Creative Commons Attribution License, which permits unrestricted use, distribution, and reproduction in any medium, provided the original work is properly cited.

\begin{abstract}
In the process of social development, there are a lot of competitions and confrontations. Participants in these competitions and confrontations always have different interests and goals. In order to achieve their goals, the participants must consider the opponent's strategy to adjust their own strategies to achieve the interests of the optimization. This is called game. Based on the definition and its stability of the passive system, the passive control items are designed to the output of the duopoly competition evolution model, and the efficacy of the control methods is shown by the Lyapunov indexes. Then, the optimal function control method is taken to carry on the chaotic anticontrol to the chaotic system, and the Lyapunov indexes illustrate the control result. At last, the chaotic game of the system is introduced by combining the chaos control and anticontrol.
\end{abstract}

\section{Introduction}

In 1980, Stutzer firstly revealed the chaos phenomenon in the Haavelmo economic growth equation [1]. Then, it has been recognized that the economic model that is based on the traditional economic theory also has inherent randomness, which makes the effects of intervention means taken by traditional ways such as the fiscal policy or the financial policy and other corresponding macrocontrol means very limited [2]. The impact and revelation of chaos theory on traditional economics have aroused the concern and research of many experts and scholars. In [3], the Cournot Nash equilibrium and the local stability were investigated based on a dynamical multiproduct Cournot duopoly model in discrete time. And the tangential bifurcation and intermittent chaos were reported by numerical simulations. In [4], the generation of chaos in economic models through exogenous shocks was investigated. And a rigorous proof of the existence of chaos in the perturbed model was given and the results shed light on a novel source of chaos in economic models. And the OGY method proposed by Ott et al. [5] has made the study of chaotic control of economic system more systematic and extensive. In [6], the OGY method was used to stabilize chaos in the macroeconomic dynamic model, and the result showed that the government can, in principle, stabilize an unstable Walrasian equilibrium in a short time by varying income tax rates or government expenditures. In [7], necessary and sufficient criteria are established to guarantee CMGPS for the fractional-order complex chaos and fractional-order real chaos based on the stability theory of fractional-order systems.

It is well known that duopoly model is one of the most representative oligopoly models. Duopoly competition is the oligopoly competition between two companies; based on the actions of the competitor, each company will take measures to maximize profits. In economics, the Cournot model and the Bertrand model are classical theories of the oligarchy, and their various extended models and deformations are used to deal with many problems in the field of industrial economics. Based on Bertrand model, the oligopolistic equilibrium price of insurance market in two cases is studied [8], one without the supply capacity limit and the other under the supply capacity limitation. Complex dynamics are shown in the study of the Cournot model [9], such as bifurcation and chaos with logarithmic demand functions. Puu [10, 11] discussed the dynamical properties of the Cournot oligarchic model and 
indicated that it will not converge to Nash equilibrium unless it is a complex trajectory. And the dynamical properties of the bounded rational duopoly model are also studied in [12].

As an extension of the Cournot duopoly model, the output duopoly competing evolution model originally developed by Agiza et al. [13] has also been further studied. Du et al. [14] used the linear stabilization method to control chaos and demonstrated a variety of results of economic chaos control. They also analyzed the effects of both the output speed adjustment and the cost function type on the results. The method can guide the unstable orbit to follow the fixed point in the area of the unstable point and then enter the stable area around the fixed point within a given period of time. The limiter method is also applied to control the chaos of the output duopoly competing evolution model [15]. The limiter method realized the control of chaos and hyperchaos by limiting the strange attractor space in chaos and hyperchaotic systems (the hyperchaotic phenomenon occurs in the nonlinear high-dimensional system, that is, at least four-dimensional nonlinear system, with multidirectional instability, while the divergence of the trajectory occurs in only one direction in chaos system [16]).

The output duopoly competing evolution model will be introduced to analysis in this paper. Boulding and Jarrett [17] proposed that the economic system has the property of the dissipative structure; namely, the economic system is open and moving away from the balance. Passive system, as a special case of dissipative system, is developed from network theory and other physical branches, and it carries out research from the energy properties of the system. It is a kind of dynamic system considering the energy exchange between the system and the outside world and can maintain the internal stability. For the system with interference, in order to stabilize the system, the feedback controller can be constructed based on the passive theory, so that the closedloop response system can remain internally stable. The system controller designed based on the passive control theory can realize the global stability of the system without singularity problem, and it has strong robustness to the change of the system parameters and the external perturbation. It is a kind of essential nonlinear control method. Because the economic system has the dissipative structure property, in this paper, we introduce the passive control method in Section 5 to conduct the chaos control to the model; the global stability of the system is achieved by constructing passive control item based on passive theory, and the effectiveness of the results can be showed by numerical simulation. In addition, in Section 6, we consider the chaotic anticontrol of the output duopoly competing evolution model on the basis of passive control, where the method used is the optimal function method [18], and its effectiveness is also validated through the numerical simulation.

The Cournot duopoly model embodies the idea of the game; that is, there are two enterprises to carry on the game of production, and each enterprise determines its own decisions based on the others' production decisions. But this game is a static game; two oligarchs make decisions at the same time, and there is no room for reaction. In [19], a dynamic duopoly game with heterogeneous players was discussed. The paper analyzed how the limiter affects the dynamics of output and the performance of players and explored the number of the equilibrium points and the distribution of conditioned equilibrium points of the model. In [20], a nonlinear Kopel duopoly game with heterogeneous players is presented to show bifurcation diagrams, phase portraits, and sensitive dependence on initial conditions by numerical simulations. In [21], the chaotic behavior of the duopoly Cournot-Puu model was controlled by introduced delayed feedback control (DFC) method and the adaptive control method to the system, and chaos was controlled during the output adjustment process in the actual economic sense. In [22], two different time delay structures for the dynamical Cournot game with two heterogeneous players were researched; the dynamics of both players output adjustments are analyzed and simulated. The paper pointed that the system may lose stability due to either a period-doubling bifurcation or a Neimark-Sacker bifurcation, but the chaotic behaviors can be stabilized by the time-delayed feedback control. In [23], a cubic utility function that was derived from a constant elasticity of substitution production function (CES) was introduced, and, based on this, a two-dimensional Cournot duopoly game with horizontal product differentiation was modeled. The stability analysis of Nash point was considered and some numerical simulations were carried out to confirm the chaotic behavior of Nash equilibrium point. Differently from the fact that authors put importance on the analysis of chaotic behavior and chaos control, in this paper, in addition to chaos control, chaos anticontrol is also considered. And considering that the static game can not respond in a timely manner, the emergence of differential game solves this problem; that is, one first makes the decision and the other determines the output according to the previous decision information and the current decision, making decision-making more effective. In this paper, this idea is applied to the chaotic game of the output duopoly competing evolution model in Sections 3 and 7.

Based on the output duopoly competing evolution model, this paper is divided into the following parts to discuss. Firstly, the fundamental theories are given in Section 2 and the basic thought of chaotic game is introduced in Section 3. Secondly, the duopoly model with its fixed point analysis is introduced in Section 4. Then, Sections 5 and 6 discuss the chaos control and the antichaos control of the system, respectively. And, finally, we consider the chaotic game of the model based on Sections 5 and 6.

\section{Fundamental Theory}

Suppose $f: C \rightarrow C$ is a map on the complex plane. If $f(\omega)=$ $\omega, \omega$ is called the fixed point of $f$ [21].

The nonlinear generalized discrete system can be written as follows [22]:

$$
\begin{aligned}
E x(k+1) & =A x(k)+f(x(k))+B \omega(k), \\
z(k) & =C x(k)+D \omega(k),
\end{aligned}
$$

where $x(k) \in R^{n}, \omega(k) \in R^{p}$, and $z(k) \in R^{p}$ represent the state, disturbed input, and output of the system, respectively; 
$f(x(k))$ is the nonlinear part of the system; $E, A \in R^{n \times n}$, $\operatorname{rank}(E)=r<n(\operatorname{rank}(T)$ means the ranks of the matrix $T) ; B, C$, and $D$ are all matrixes with appropriate dimension.

Suppose $(E, A)$ is regular, causal, and stable [23], so the Lyapunov equation [23] is

$$
A^{T} V A-E^{T} V E=-E^{T} W E .
$$

And for every $W>0(W>0$ means that $W$ is positive definite), we have the only positive semidefinite solution $V$ which satisfies the equation $\operatorname{rank}\left(E^{T} V E\right)=\operatorname{rank}(E)=r$.

Definition 1 (see [22]). For system (1), if there is a nonnegative definite function $V(x(k))$ meeting the following inequality for any $k \geq 0$ and any input item $\omega(k)$,

$$
V(x(k+1))-V(x(k)) \leq z^{T} \omega(k),
$$

then system (1) is called to be passive.

Without the input and output, system (1) is

$$
E x(k+1)=A x(k)+f(x(k)) .
$$

For system (4), it is assumed that for any satisfying compatible initial condition $E x(0)=E x_{0}$, the system has only one solution $x(k)$.

Consider the following nonlinear discrete controlled system

$$
E x(k+1)=A x(k)+f(x(k))+B_{1} u(k),
$$

where $u(k) \in R^{m}$ is the control item and $B_{1}$ is the arbitrary matrix with appropriate dimension.

The aim is looking for a passive state feedback controller $u(k)=G x(k)$ which can make the system

$$
E x(k+1)=\left(A+B_{1} G\right) x(k)+f(x(k))
$$

stable.

According to the Lyapunov stability theory, (6) is $E$ asymptotically stable [24]; that is, $\lim _{k \rightarrow 0} E x(k)=0$; then it can be proved that $\lim _{k \rightarrow 0} x(k)=0$ [23], so, we can say that (6) has asymptotic stability of zero solution and is a passive system.

Lemma 2 (see [22]). For any $n$ dimensional vectors $x$ and $y$, there is $2 x^{T} y \leq x^{T} x+y^{T} y$.

There are results as follows.

Theorem 3 (see [22]). If system (4) meets the following conditions:

(1) $\|f(x(k))\| \leq \rho\|E x(k)\|, \rho>0$ is a constant;

(2) $\|A x(k)\| \leq \gamma\|E x(k)\|, \gamma>0$ is a constant;

(3) $W-2 \gamma \rho \lambda_{\max }(V) I-\rho^{2} \lambda_{\max } I>0$, where $W, V$ are matrixes that meet (2) and $\lambda_{\max }(M)$ is the biggest eigenvalue of $M$, then system (4) has asymptotic stability of zero solution.
Theorem 4 (see [22]). Suppose there is a constant $\varepsilon>0$ which can make system (5) have

(1) $\|f(x(k))\| \leq \rho\|E x(k)\|, \rho>0$ is a constant;

(2) $\|A x(k)\| \leq \gamma\|E x(k)\|, \gamma>0$ is a constant;

(3) $S<0$, where

$$
\begin{aligned}
S= & -W+2 \gamma \rho \lambda_{\max }(V) I+\rho^{2} \lambda_{\max } I \\
& +\frac{\varepsilon^{2} \gamma^{2}}{4} \lambda_{\max }\left(V B_{1} B_{1}^{T}\left(V^{2}+V\right) B_{1} B_{1}^{T} V\right) I
\end{aligned}
$$

$-\varepsilon \gamma^{2} \lambda_{\max }\left(V B_{1} B_{1}^{T} V\right) I$, and $W, V$ are matrixes that meet (2); $\lambda_{\max }(M)$ is the biggest eigenvalue of $M$.

Then system (5) has a passive state feedback controller that can make (6) have asymptotic stability of zero solution and be passive. And the controller is

$$
u(k)=-\frac{\varepsilon}{2} B_{1}^{T} V A x(k) .
$$

The basic characteristic of chaos movement is that the movement is extremely sensitive to initial conditions [24]. And Lyapunov index is the quantitative index that can describe this phenomenon. For one dimensional mapping

$$
x_{n+1}=f\left(x_{n}\right) \text {. }
$$

Consider the initial value $x_{0}$ and the adjacent value $x_{0}+\delta x_{0}$. After one iteration, the distance between two points is

$$
\delta x_{1}=\left|f\left(x_{0}+\delta x_{0}\right)-f\left(x_{0}\right)\right|=\frac{d f\left(x_{0}\right)}{d x} \delta x_{0} .
$$

After $n$ iterations, the distance between two points is

$$
\begin{aligned}
\delta x_{n} & =\left|f^{(n)}\left(x_{0}+\delta x_{0}\right)-f^{(n)}\left(x_{0}\right)\right|=\frac{d f^{(n)}\left(x_{0}\right)}{d x} \delta x_{0} \\
& =e^{L E * n} \delta x_{0} .
\end{aligned}
$$

Take $n \rightarrow \infty$; there is

$$
L E=\lim _{n \rightarrow \infty} \frac{1}{n} \ln \frac{\delta x_{n}}{\delta x_{0}}=\lim _{n \rightarrow \infty} \frac{1}{n} \ln \left|\frac{d f^{(n)}\left(x_{0}\right)}{d x} \delta x_{0}\right| .
$$

$L E$ represents the separated index caused by each iteration on average of the distance between two points.

\section{Basic Thought of Chaotic Game}

Consider $m$-dimensional discrete chaotic system

$$
w_{n+1}=G\left(w_{n}\right)
$$

where $w_{n}=\left(x_{1}(n), x_{2}(n), \ldots, x_{m}(n)\right)^{T}$ is the state of the system at stage $n$. When parameters of (13) are within a certain range, (13) exhibits chaotic behavior. Chaos control is to make system stable, while anticontrol is to enhance chaos.

In the following parts, we will discuss the chaos control and anticontrol. And then, on the basis of the theory of 
differential game, we will discuss the chaos game of system (13).

Consider

$$
w_{n+1}=G\left(w_{n}\right)+p_{n}+q_{n},
$$

where $p_{n}=p\left(w_{n}, p_{1}, q_{1}, \ldots, p_{n-1}, q_{n-1}\right), q_{n}=q\left(w_{n}, p_{1}, q_{1}\right.$, $\left.\ldots, p_{n-1}, q_{n-1}\right)$ are controllers needing to be designed. $p_{n}$ is for stability and $q_{n}$ for chaos. Specific steps are as follows.

When $n=1, w_{2}=G\left(w_{1}\right)+p_{1}+q_{1} \cdot p_{1}=p\left(w_{1}\right)$ is the first item that we need to design for stability, which is related to $w_{1} \cdot q_{1}=q\left(w_{1}, p_{1}\right)$ is the first item we will design for chaos after we got $p_{1}=p\left(w_{1}\right)$.

When $n=2, w_{3}=G\left(w_{2}\right)+p_{2}+q_{2}$. We can know that $w_{3}$ is associated with $G\left(w_{2}\right)$, while $G\left(w_{2}\right)$ is associated with $p_{1}$, $q_{1}$, so the selection of $p_{2}$ is related to $p_{1}, q_{1}$, and the selection of $q_{2}$ needs to consider $p_{1}, q_{1}$, and $p_{2}$.

For the $n-1$ step, $w_{n}=G\left(w_{n-1}\right)+p_{n-1}+q_{n-1} \cdot w_{n}$ is associated with $G\left(w_{n-1}\right)$, and $G\left(w_{n-1}\right)$ is associated with $p_{n-2}$, $q_{n-2}$. Considering stability and chaos game, the selection of $p_{n-2}$ is related to $p_{n-3}, q_{n-3}$, and the selection of $q_{n-2}$ needs to consider $p_{n-3}, q_{n-3}$, and $p_{n-2}$. Therefore, $p_{n-1}$ is related to $p_{1}$, $q_{1}, \ldots, p_{n-2}, q_{n-2}$, and $q_{n-1}$ is related to $p_{1}, q_{1}, \ldots, p_{n-2}, q_{n-2}$, $p_{n-1}$.

Based on the above ideas, the selection of control items in the game will be discussed as follows.

\section{The Output Duopoly Competing Evolution Model with Its Fixed Point Analysis}

Consider the model as follows [13]:

$$
\begin{aligned}
& q_{1}(t+1)=q_{1}(t)+\alpha_{1} q_{1}(t) \\
& \cdot {\left[a-b\left(q_{1}(t)+q_{2}(t)\right)-\left(b+2 e_{1}\right) q_{1}(t)-d_{1}\right] } \\
& \cdot(1-s), \\
& q_{2}(t+1)=q_{2}(t)+\alpha_{2} q_{2}(t) \\
& \cdot\left[a-b\left(q_{1}(t)+q_{2}(t)\right)-\left(b+2 e_{2}\right) q_{2}(t)-d_{2}\right] \\
& \cdot(1-s),
\end{aligned}
$$

where $q_{1}(t)$ and $q_{2}(t)$ are the output of two companies at time $t$, respectively, $t=1,2, \ldots ; a, b$ are positive constants, and $a$ is the highest price of the market; assume that the cost functions are $C\left(q_{1}\right)=c_{1}+d_{1} q_{1}+e_{1} q_{1}{ }^{2}$ and $C\left(q_{2}\right)=c_{2}+d_{2} q_{2}+e_{2} q_{2}{ }^{2}$, and the first derivatives of $C\left(q_{1}\right)$ and $C\left(q_{2}\right)$ are positive and the second derivatives are negative, so we can get $d_{i}, e_{i}>0$; since companies' marginal profits are demanded to be less than the price of the same product in market, we have $d_{1}+2 e_{1} x<$ $a, d_{2}+2 e_{2} y<a ; \alpha_{i}$ is positive parameters, which shows the company's adjustment speed.

$$
\begin{aligned}
& Q\left(q_{1}, q_{2}\right)=q_{1} \\
& \quad+\alpha_{1} q_{1}\left[a-b\left(q_{1}+q_{2}\right)-\left(b+2 e_{1}\right) q_{1}-d_{1}\right](1-s), \\
& R\left(q_{1}, q_{2}\right)=q_{2} \\
& \quad+\alpha_{2} q_{2}\left[a-b\left(q_{1}+q_{2}\right)-\left(b+2 e_{2}\right) q_{2}-d_{2}\right](1-s),
\end{aligned}
$$

assuming that the fixed point of model is $\left(q_{1}{ }^{*}, q_{2}{ }^{*}\right)$; according to definition, we have

$$
\begin{aligned}
& Q\left(q_{1}{ }^{*}, q_{2}{ }^{*}\right)=q_{1}{ }^{*}, \\
& R\left(q_{1}{ }^{*}, q_{2}{ }^{*}\right)=q_{2}{ }^{*} .
\end{aligned}
$$

The equations' solutions have the following several cases:

(1) $\left(q_{1}{ }^{*}, q_{2}{ }^{*}\right)=(0,0)$;

(2) $\left(q_{1}{ }^{*}, q_{2}{ }^{*}\right)=\left(0,\left(a-d_{2}\right) / 2\left(b+e_{2}\right)\right)$;

(3) $\left(q_{1}{ }^{*}, q_{2}{ }^{*}\right)=\left(\left(a-d_{1}\right) / 2\left(b+e_{1}\right), 0\right)$;

(4) $\left(q_{1}{ }^{*}, q_{2}{ }^{*}\right)=\left(\left(\left(a-d_{1}\right)\left(2 b+2 e_{2}\right)-b\left(a-d_{2}\right)\right) /\left(3 b^{2}+4 b e_{1}+\right.\right.$ $\left.4 b e_{2}+4 e_{1} e_{2}\right),\left(\left(a-d_{2}\right)\left(2 b+2 e_{1}\right)-b\left(a-d_{1}\right)\right) /\left(3 b^{2}+\right.$ $\left.\left.4 b e_{1}+4 b e_{2}+4 e_{1} e_{2}\right)\right)$.

In this article, we just consider the fixed point that both $q_{1}{ }^{*}$ and $q_{2}{ }^{*}$ are not zero.

\section{Chaos Control of the Output Duopoly Competing Evolution Model}

In order to facilitate the following analysis, the system (15) needs to be rewritten into the form of system (4). If we directly change system (15) into (4), there is

$$
E_{0} x(k+1)=A_{0} x(k)+f_{0}(x(k))
$$

where $E_{0}=\left(\begin{array}{cc}1 & 0 \\ 0 & 1\end{array}\right), A_{0}=\left(\begin{array}{cc}a_{11} & a_{12} \\ a_{21} & a_{22}\end{array}\right), f_{0}(x(k))=\left(\begin{array}{l}f_{1}(x(k)) \\ \left.f_{2}(x(k))\right)\end{array}\right), a_{11}$, $a_{12}, a_{21}, a_{22}, f_{1}(x(k))$, and $f_{2}(x(k))$ will be given in the following discussion. It is quite clear that $E_{0}$ cannot meet the requirement that $\operatorname{rank}(E)=r<n$; here, $n=2$. So, a function $q_{3}(t)$ is tried to be introduced into system (15), which meets the following equation:

$$
0 * q_{3}(t+1)=q_{3}(t)(* \text { means multiplication }) .
$$

We have variable substitution

$$
\begin{aligned}
x^{*} & =q_{1}^{*} ; \\
y^{*} & =q_{2}^{*}, \\
x_{1}(t) & =q_{1}(t)-x^{*} ; \\
x_{2}(t) & =q_{2}(t)-y^{*} ; \\
x_{3}(t) & =q_{3}(t) .
\end{aligned}
$$

So, system (15) can be written as

$$
E x(k+1)=A x(k)+f(x(k)),
$$


where

$$
\begin{aligned}
& E=\left(\begin{array}{lll}
1 & 0 & 0 \\
0 & 1 & 0 \\
0 & 0 & 0
\end{array}\right), \\
& x(k)=\left(\begin{array}{c}
x_{1}(k) \\
x_{2}(k) \\
x_{3}(k)
\end{array}\right), \\
& A=\left(\begin{array}{ccc}
a_{11} & a_{12} & 0 \\
a_{21} & a_{22} & 0 \\
0 & 0 & 1
\end{array}\right), \\
& f(x(k))=\left(\begin{array}{l}
f_{1}(x(k)) \\
f_{2}(x(k)) \\
f_{3}(x(k))
\end{array}\right), \\
& a_{11}=1+\alpha_{1} a(1-s)-b \alpha_{1}(1-s)\left(x^{*}+y^{*}\right)-a \alpha_{1}(b \\
& \left.+2 e_{1}\right)(1-s) x^{*}-\alpha_{1} d_{1}(1-s)-\alpha_{1} x^{*} b(1-s) ; \\
& a_{12}=-\alpha_{1} b x^{*}(1-s) \text {; } \\
& a_{21}=-\alpha_{2} b y^{*}(1-s) \text {; } \\
& a_{22}=1+\alpha_{2} a(1-s)-b \alpha_{2}(1-s)\left(x^{*}+y^{*}\right)-a \alpha_{2}(b \\
& \left.+2 e_{2}\right)(1-s) x^{*}-\alpha_{2} d_{2}(1-s)-\alpha_{2} x^{*} b(1-s) ; \\
& f_{1}(x(k))=-\left[\alpha_{1} b(1-s) x_{1}(k) x_{2}(k)\right. \\
& +\left(\alpha_{1} b(1-s)+\alpha_{1}\left(b+2 e_{1}\right)(1-s)\right) x_{1}^{2}(t) \\
& +\alpha_{1} b(1-s) x^{*}\left(x^{*}+y^{*}\right) \\
& \left.+\alpha_{1}\left(b+2 e_{1}\right)(1-s) x^{* 2}+\alpha_{1} d_{1}(1-s) x^{*}\right] ; \\
& f_{2}(x(k))=-\left[\alpha_{2} b(1-s) x_{1}(k) x_{2}(k)\right. \\
& +\left(\alpha_{2} b(1-s)+\alpha_{2}\left(b+2 e_{2}\right)(1-s)\right) x_{2}^{2}(t) \\
& +\alpha_{2} b(1-s) y^{*}\left(x^{*}+y^{*}\right) \\
& \left.+\alpha_{2}\left(b+2 e_{2}\right)(1-s) y^{* 2}+\alpha_{2} d_{2}(1-s) y^{*}\right] ; \\
& f_{3}(x(k))=0 .
\end{aligned}
$$

Here, $\operatorname{rank}(E)=r<n$, where $\operatorname{rank}(E)=2<n=3$. Therefore, the fixed point of system $(21)$ is $(0,0,0)$.

According to the content of passive control in the basic theory, we can get the following theorem.

Theorem 5. Suppose there is a constant $\varepsilon>0$ which makes system (5) have

(1) $\|f(x(k))\| \leq \rho\|E x(k)\|, \rho>0$ is a constant;

(2) $\|A x(k)\| \leq \gamma\|E x(k)\|, \gamma>0$ is a constant;
(3) $S<0$, where

$$
\begin{aligned}
S= & -W+2 \gamma \rho \lambda_{\max }(V) I+\rho^{2} \lambda_{\max } I \\
& +\frac{\varepsilon^{2}}{4} \lambda_{\max }\left(V\left(V^{2}+V\right) V\right) I-\varepsilon \gamma^{2} \lambda_{\max }(V V) I \\
& -\varepsilon \rho \gamma \lambda_{\max }(V V) I ;
\end{aligned}
$$

and $W, V$ are matrixes that meet (2); $\lambda_{\max }(M)$ is the biggest eigenvalue of $M$.

Then system (5) has a passive state feedback controller that can make (6) have stability of zero solutions and be passive. And the controller is

$$
u(k)=-\frac{\varepsilon}{2} B_{1}^{T} V A x(k) .
$$

According to conditions of Theorem 5, a control item is introduced to (21)

$$
u(k)=-\frac{\varepsilon}{2} B_{1}^{T} V A x(k) .
$$

Due to the arbitrariness of matrix $B_{1}$, let $B_{1}=I$. So the controlled system is

$$
E x(k+1)=A x(k)+f(x(k))-\frac{\varepsilon}{2} V A x(k) .
$$

We will prove that the system (26) has stability of zero solutions and (26) is passive.

Proof. The generalized Lyapunov function of (6) is selected as $V(E x(k))=(E x(k))^{T} V(E x(k))$. And according to the conditions of the Lemma and Theorem 5 , there is

$$
\begin{aligned}
\Delta V & (E x(k))=V(E x(k+1))-V(E x(k)) \\
& =((A+G) x(k)+f(x(k)))^{T} \\
& \cdot V((A+G) x(k)+f(x(k)))-(E x(k))^{T} \\
& \cdot V(E x(k))=-(E x(k))^{T} W(E x(k)) \\
& +2(A x(k))^{T} V(G x(k))+2(A x(k))^{T} V f \\
& +(G x(k))^{T} V(G x(k))+f(x(k))^{T} V f(x(k)) .
\end{aligned}
$$

According to (16), we have $G=-(\varepsilon / 2) V A$, so

$$
\begin{aligned}
& \Delta V(E x(k)) \leq(E x(k))^{T}\left(-W+2 \gamma \rho \lambda_{\max }(V) I\right. \\
& +\rho^{2} \lambda_{\max } I+\frac{\varepsilon^{2}}{4} \lambda_{\max }\left(V^{T} V V\right) I-\varepsilon \gamma^{2} \lambda_{\max }(V V) I \\
& \left.\quad-\varepsilon \rho \gamma \lambda_{\max }(V V) I\right) .
\end{aligned}
$$

Let

$$
\begin{aligned}
S= & -W+2 \gamma \rho \lambda_{\max }(V) I+\rho^{2} \lambda_{\max } I \\
& +\frac{\varepsilon^{2}}{4} \lambda_{\max }\left(V^{T} V V\right) I-\varepsilon \gamma^{2} \lambda_{\max }(V V) I \\
& -\varepsilon \rho \gamma \lambda_{\max }(V V) I .
\end{aligned}
$$


So

$$
\Delta V(E x(k)) \leq(E x(k))^{T} S(E x(k)) .
$$

According to Theorems 3, and 4 and the conditions of Theorem 5, (5) has stability of zero solutions.

Because the model does not have any input and output except the control item $u(k)$, and $\omega(k)$ is arbitrary, so we choose $\omega(k)=0$. Then we just need to prove

$$
V(E x(k+1))-V(E x(k)) \leq 0 .
$$

The generalized Lyapunov function described above is the nonnegative definite function required in the definition. Thus, according to the conditions of the theorem and the proof above, we have

$$
V(E x(k+1))-V(E x(k)) \leq 0 .
$$

Therefore, system (6) is passive.

For example, we take the parameters in (15) as $a=10$, $b=1, d_{1}=1, d_{2}=1, e_{1}=1, e_{2}=1.1, s=0.3, \alpha_{1}=0.52$, and $\alpha_{2}=0.1$. Then the fixed point is $\left(x^{*}, y^{*}\right)=(1.8228,1.7089)$. For these parameters, we can get Lyapunov indexes of (15) as 0.2540 and -1.4579 . Because $0.2540>0$, system (15) under parameters above is chaotic.

Under the parameters, we have,

$$
E=\left(\begin{array}{lll}
1 & 0 & 0 \\
0 & 1 & 0 \\
0 & 0 & 0
\end{array}\right)
$$

$$
\begin{aligned}
A & =\left(\begin{array}{ccc}
-1.654 & -0.6635 & 0 \\
-0.1196 & 0.4976 & 0 \\
0 & 0 & 1
\end{array}\right), \\
f(x(k)) & =\left(\begin{array}{l}
f_{1}(x(k)) \\
f_{2}(x(k)) \\
f_{3}(x(k))
\end{array}\right)
\end{aligned}
$$

where $f_{1}(x(k))=-\left[0.364 x_{1}(k) x_{2}(k) 1.456 x_{1}^{2}(t)+6.2281\right]$, $f_{2}(x(k))=-\left[0.07 x_{1}(k) x_{2}(k)+0.294 x_{2}^{2}(t)+1.1963\right]$, $f_{3}(x(k))=0$, and the fixed point is $(0,0,0)$.

When $S_{0} \neq 4.276$ and $S_{0} \neq 1.63$, we can get $\left|S_{0} E-A\right| \neq 0$; then we can say that $(E, A)$ is regular [25].

According to the fundamental theory, $W$ is arbitrary and we take $W$ as

$$
W=\left(\begin{array}{lll}
0 & 1 & 0 \\
1 & 1 & 0 \\
0 & 0 & 1
\end{array}\right)
$$

bring $A, E$, and $W$ into (2); then we have the only positive semidefinite solution

$$
V=\left(\begin{array}{ccc}
0.8473 & -0.1147 & 0 \\
-0.1147 & 0.4724 & 0 \\
0 & 0 & 0
\end{array}\right)
$$

In an actual market of oligarch competition, the output of participates in the competition is not infinite because the economic resources are always limited. Therefore, we assume that production is bounded. Then there is an $M>0$ satisfying $\left|x_{1}(k)\right|<M,\left|x_{2}(k)\right|<M,\left|x_{3}(k)\right|<M$.

And

$$
\begin{aligned}
\|\operatorname{Ex}(k)\| & =\sqrt{x_{1}^{2}(k)+x_{2}^{2}(k),} \\
\|A x(k)\| & =\sqrt{\left(1.654 x_{1}(k)+0.6635 x_{2}(k)\right)^{2}+\left(-0.1196 x_{1}(k)+0.4976 x_{2}(k)\right)^{2}+x_{3}^{2}(k),} \\
\|f(x(k))\| & =\sqrt{\left(0.364 x_{1}(t) x_{2}(t)+1.456 x_{1}^{2}(t)+6.2281\right)^{2}+\left(0.07 x_{1}(t) x_{2}(t)+0.294 x_{2}^{2}(t)+1.1963\right)^{2}} .
\end{aligned}
$$

Because production is limited, we can get $\|A(x(k))\| \leq$ $\gamma\|E x(k)\|$ when $\gamma>1.3594$ and when $\rho$ is big enough, we have $\rho \sqrt{2} M>\sqrt{3.4427 M^{4}+23.5337 M^{2}+40.2203}$.

Then the controlled system is

$$
\begin{aligned}
& x_{1}(t+1) \\
&=-1.654 x_{1}(t)-0.6635 x_{2}(t) \\
&-\left(0.364 x_{1}(t) x_{2}(t)+1.456 x_{1}^{2}(t)+6.2281\right) \\
&+\frac{\varepsilon}{2}\left(1.3877 x_{1}(t)+0.6193 x_{2}(t)\right),
\end{aligned}
$$

$$
\begin{aligned}
& x_{2}(t+1) \\
&=-0.1196 x_{1}(t)+0.4976 x_{2}(t) \\
&-\left(0.07 x_{1}(t) x_{2}(t)+0.294 x_{2}^{2}(t)+1.1963\right) \\
&-\frac{\varepsilon}{2}\left(0.1332 x_{1}(t)+0.3112 x_{2}(t)\right) .
\end{aligned}
$$

The bifurcation figure is given with the change of the parameter $\alpha$. It can be seen from the Figure 1 that when $\alpha$ is taken as 0.476 , the system output bifurcation occurs. When 


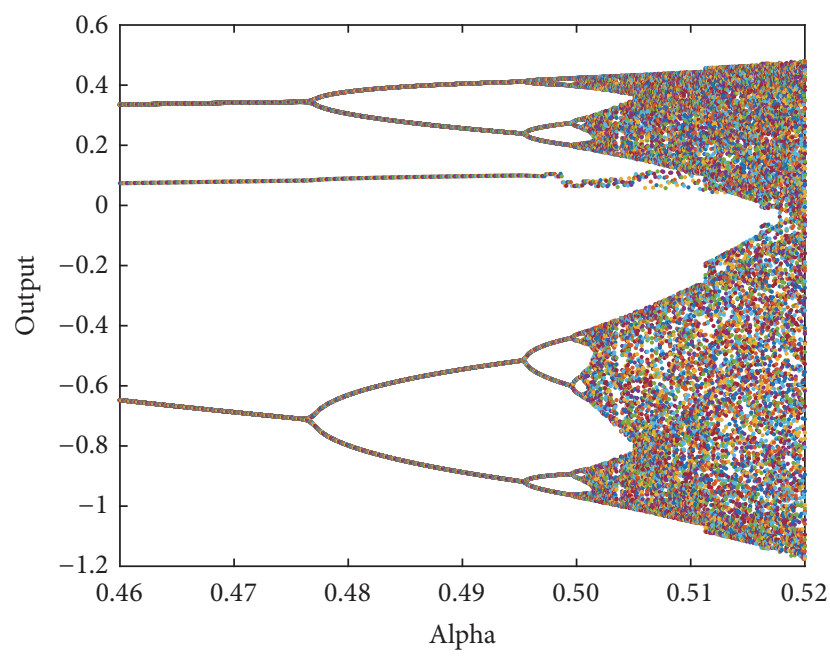

FIGURE 1: The bifurcation of the output with the change of $\alpha$.

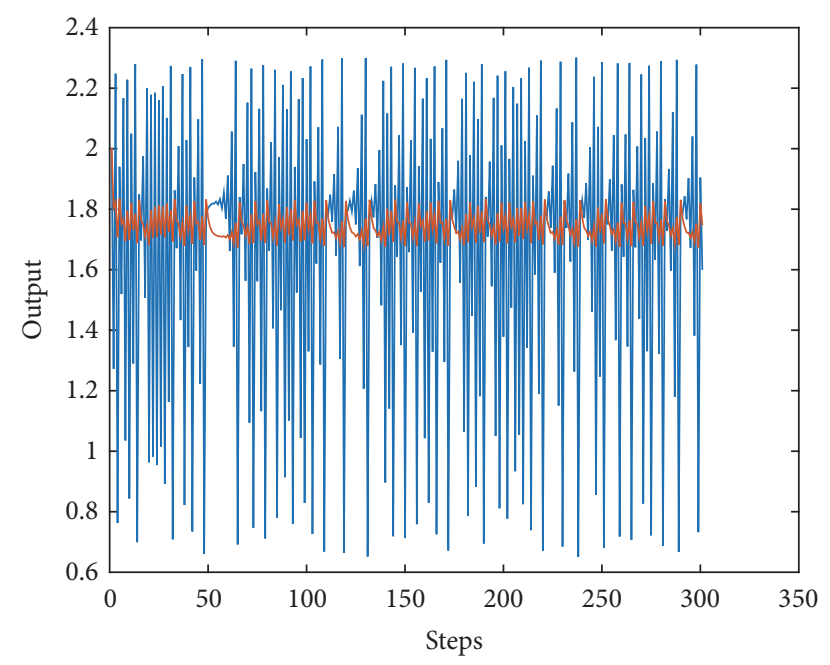

FIGURE 2: Original output of system.

$\alpha$ is 0.5 , the system output shows obvious chaos. Then the output change of the system when $\alpha$ is 0.52 will be given.

The blue curve represents the output of $x$, and the red one represents $y$.

It can be seen from Figure 2 that, under the given parameters in this example, the output of the system exhibits an irregular increase or decrease with the number of iterations increases; that is, the fixed point of the system (15) is unstable, and the Lyapunov indexes of the system can also show this.

It can be seen from Figure 3 that, after the introduction of passive controller, with the change of control coefficient, the output change gradually becomes regular under the increase of the iterations, and finally the system achieves global stability. The output of the system is stabilized to the fixed point, which means that the enterprises' output is no longer unpredictable and confusing. With this control item, the output trends to be stable, and enterprises can control their output to avoid wasting of resource. As can be seen from the figure, with the increase of the control coefficient, the output stabilizes to the fixed point faster and faster. In Figure 3(c), the control system needs about 50 iterations to stabilize to the fixed point, while, in Figure 3(d), the system just needs 25 iterations.

Under the selected parameters above, the corresponding Lyapunov indexes of control system are as follows

(1) $\varepsilon=0.8$, Lyapunov indexes are 0.3477 and -0.4531 ;

(2) $\varepsilon=1$, Lyapunov indexes are 0.0421 and -0.1474 ;

(3) $\varepsilon=1.2$, Lyapunov indexes are -0.0526 and -0.0527 ;

(4) $\varepsilon=1.4$, Lyapunov indexes are -0.0526 and -0.0527 .

The change of Lyapunov indexes of the control system with $\varepsilon$ is shown in Figure 4. The red curve represents the Lyapunov index of $x$, and the green one represents $y$.

Figure 4 shows the change of Lyapunov indexes with $\varepsilon$, and, with the change of $\varepsilon$, the Lyapunov index of $x$ is getting smaller and $y$ 's is getting bigger, but finally the Lyapunov indexes of two variables are negative. This shows the effectiveness of passive control.

Figure 5 shows the system output with the change of the control parameter $\varepsilon$. It can be seen from Figure 5 when $\varepsilon$ is bigger than 1 that the output is no longer showing the case of bifurcation but is stable at the same value. The results of Figures 5 and 4 are in agreement, and this shows the effectiveness of the control.

\section{The Chaos Anticontrol of the Output Duopoly Competing Evolution Model}

Zero solutions of system (26) are stable and the passivity has been proved. Now the optimal function control method [17] is taken to conduct the chaos anticontrol of (26). Equation (26) can be written in the following form, and we just consider $x_{1}$ and $x_{2}$ :

$$
\begin{aligned}
& x_{1}(n+1)=x_{1}(n)+F\left(x_{1}(n), x_{2}(n), x_{3}(n)\right), \\
& x_{2}(n+1)=x_{2}(n)+G\left(x_{1}(n), x_{2}(n), x_{3}(n)\right),
\end{aligned}
$$

where

$$
\begin{aligned}
& F\left(x_{1}(n), x_{2}(n), x_{3}(n)\right)=a_{11} x_{1}(n)+a_{12} x_{2}(n) \\
& \quad+f_{1}\left(x_{1}(n), x_{2}(n)\right)-\frac{\varepsilon}{2}\left[\left(a_{11} V_{11}+a_{21} V_{12}\right) x_{1}(n)\right. \\
& \left.\quad+\left(a_{12} V_{11}+a_{22} V_{12}\right) x_{2}(n)+V_{13} x_{3}(n)\right], \\
& G\left(x_{1}(n), x_{2}(n), x_{3}(n)\right)=a_{21} x_{1}(n)+a_{22} x_{2}(n) \\
& \quad+f_{2}\left(x_{1}(n), x_{2}(n)\right)-\frac{\varepsilon}{2}\left[\left(a_{11} V_{21}+a_{21} V_{22}\right) x_{1}(n)\right. \\
& \left.\quad+\left(a_{12} V_{21}+a_{22} V_{22}\right) x_{2}(n)+V_{23} x_{3}(n)\right],
\end{aligned}
$$




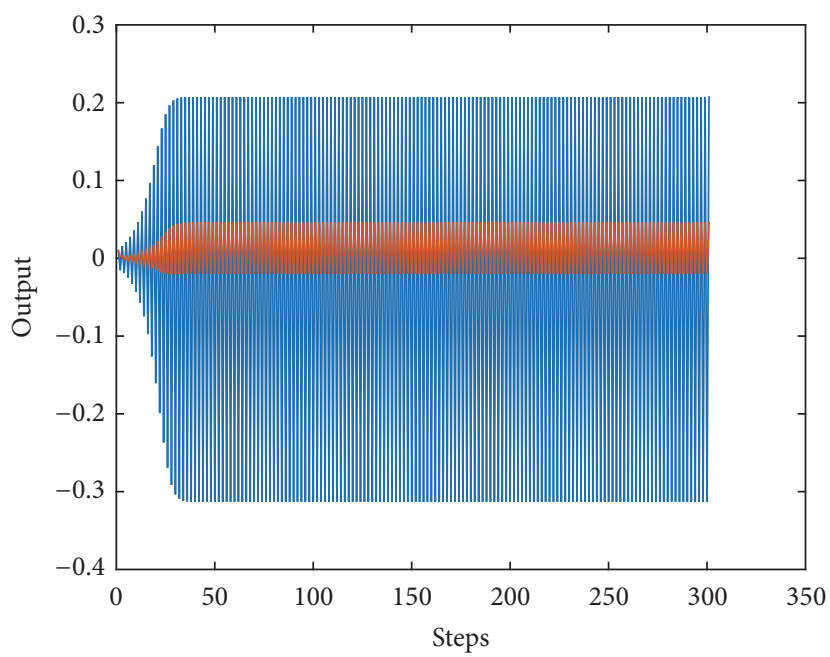

(a)

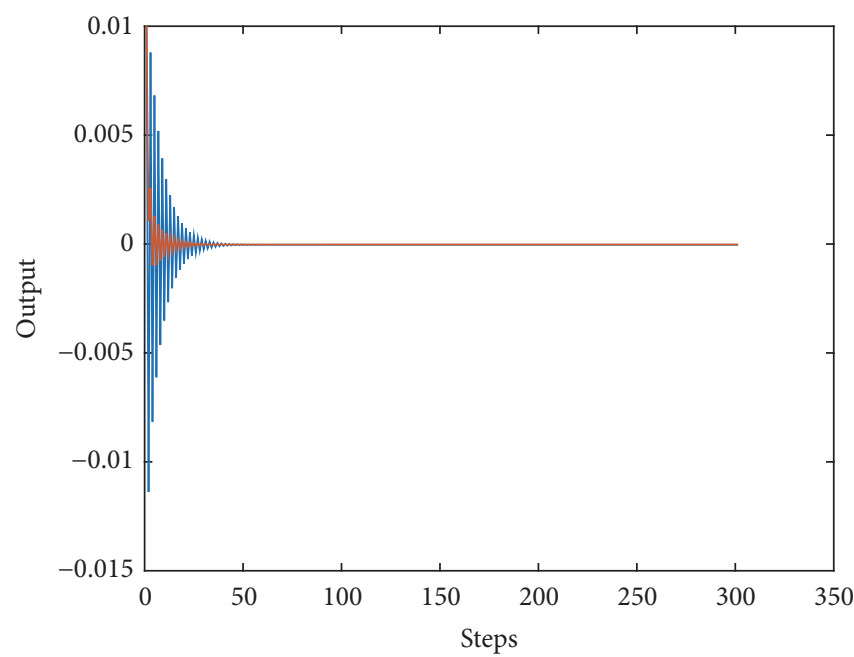

(c)

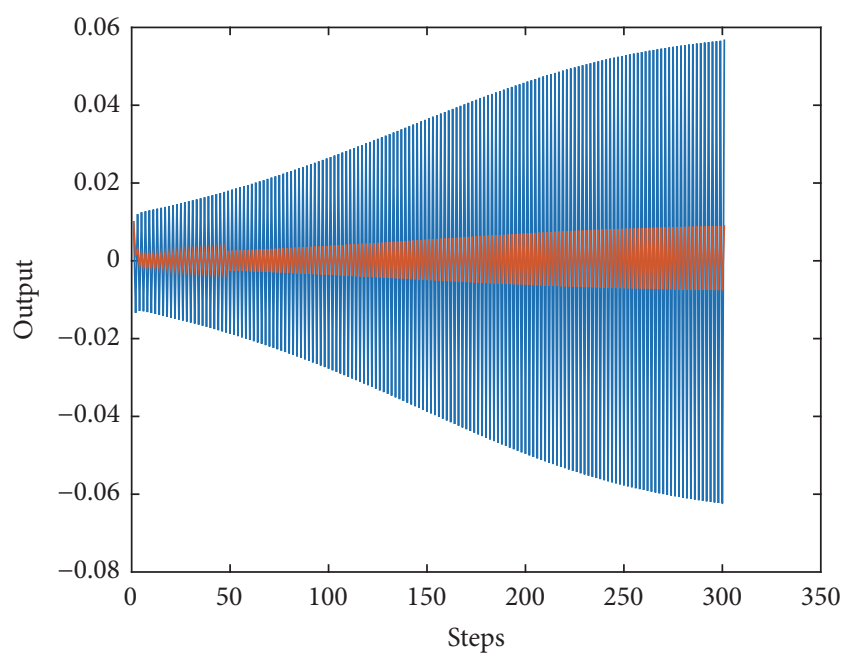

(b)

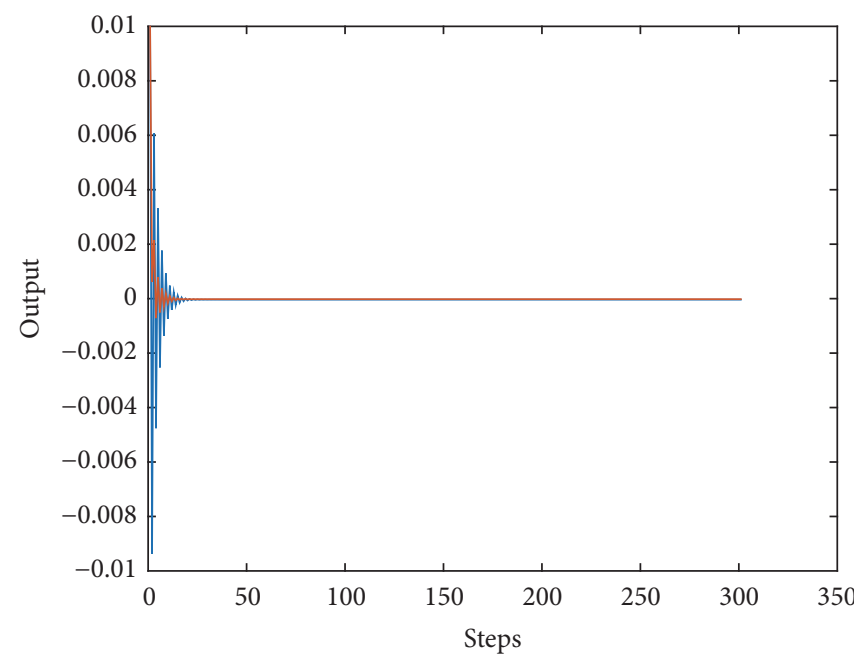

(d)

FIGURE 3: The changing of the output of the controlled system (a) $\varepsilon=0.8$; (b) $\varepsilon=1$; (c) $\varepsilon=1.2$; (d) $\varepsilon=1.4$.

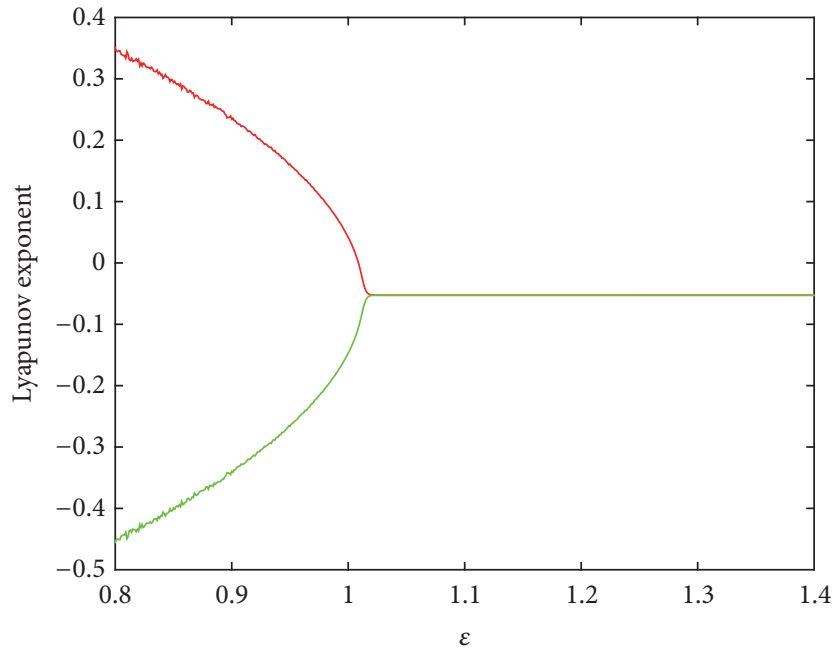

FIGURE 4: The change of Lyapunov indexes with $\varepsilon$.

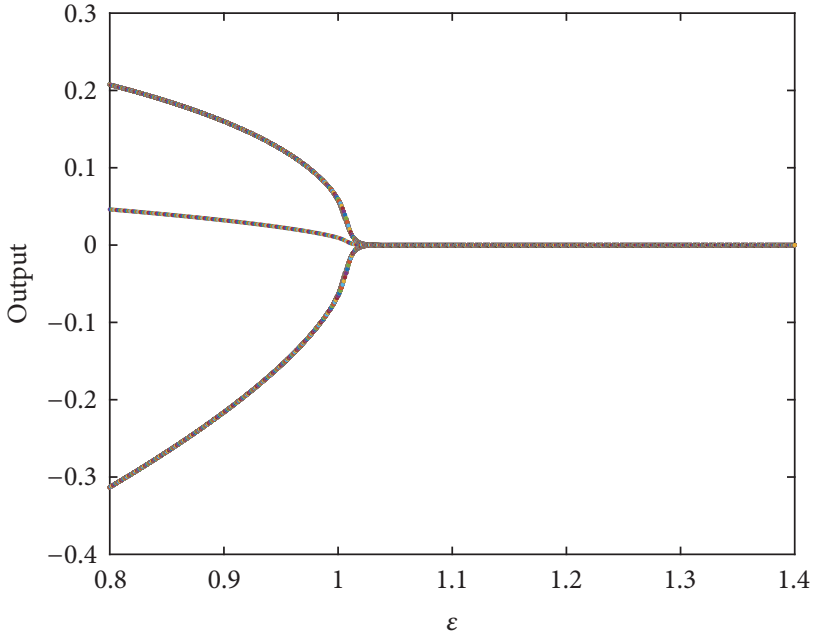

FIgURE 5: The bifurcation of the output with the change of $\varepsilon$. 


$$
\begin{aligned}
& f_{1}\left(x_{1}(n), x_{2}(n)\right)=-\left[\alpha_{1} b(1-s) x_{1}(n) x_{2}(n)\right. \\
& \quad+\left(\alpha_{1} b(1-s)+\alpha_{1}\left(b+2 e_{1}\right)(1-s)\right) x_{1}^{2}(n) \\
& \quad+\alpha_{1} b(1-s) x^{*}\left(x^{*}+y^{*}\right) \\
& \left.\quad+\alpha_{1}\left(b+2 e_{1}\right)(1-s) x^{* 2}+\alpha_{1} d_{1}(1-s) x^{*}\right], \\
& f_{2}\left(x_{1}(n), x_{2}(n)\right)=-\left[\alpha_{2} b(1-s) x_{1}(n) x_{2}(n)\right. \\
& \quad+\left(\alpha_{2} b(1-s)+\alpha_{2}\left(b+2 e_{2}\right)(1-s)\right) x_{2}^{2}(n) \\
& \quad+\alpha_{2} b(1-s) y^{*}\left(x^{*}+y^{*}\right) \\
& \left.\quad+\alpha_{2}\left(b+2 e_{2}\right)(1-s) y^{* 2}+\alpha_{2} d_{2}(1-s) y^{*}\right]
\end{aligned}
$$

and $a_{11}, a_{12}, a_{21}$, and $a_{22}$ have been given in the fifth part, and $V_{11}, V_{12}, V_{21}, V_{22}$ are the corresponding elements of $V$.

We consider the following anticontrol system:

$$
\begin{aligned}
& x_{1}(n+1) \\
& =x_{1}(n)+F\left(x_{1}(n), x_{2}(n), x_{3}(n)\right) \\
& \quad+k\left(x_{1}(n)+F\left(x_{1}(n), x_{2}(n), x_{3}(n)\right)-x_{1}(n)\right), \\
& x_{2}(n+1) \\
& =x_{2}(n)+G\left(x_{1}(n), x_{2}(n), x_{3}(n)\right) \\
& \quad+k\left(x_{2}(n)+G\left(x_{1}(n), x_{2}(n), x_{3}(n)\right)-x_{2}(n)\right) .
\end{aligned}
$$

For example, we take the parameters in (40) as $a=10, b=1$, $d_{1}=1, d_{2}=1, e_{1}=1, e_{2}=1.1, s=0.3 \alpha_{1}=0.52$, and $\alpha_{2}=0.1$. According to the previous example, we can get the corresponding anticontrol system

$$
\begin{aligned}
& x_{1}(n+1)=x_{1}(n)-(k+1)\left[2.654 x_{1}(n)\right. \\
& \quad+0.6635 x_{2}(n) \\
& \quad+\left(0.364 x_{1}(n) x_{2}(n)+1.456 x_{1}^{2}(n)+6.2281\right) \\
& \left.\quad-\frac{\varepsilon}{2}\left(1.3877 x_{1}(n)+0.6193 x_{2}(n)\right)\right], \\
& x_{2}(n+1)=x_{2}(n)-(k+1)\left[1.1196 x_{1}(n)\right. \\
& \quad-0.4976 x_{2}(n) \\
& \quad+\left(0.07 x_{1}(n) x_{2}(n)+0.294 x_{2}^{2}(n)+1.1963\right) \\
& \left.\quad+\frac{\varepsilon}{2}\left(0.1332 x_{1}(n)+0.3112 x_{2}(n)\right)\right]
\end{aligned}
$$

where $\varepsilon=1.1$.

The change of the output with the change of steps under the parameters is shown in Figure 6.

The Lyapunov indexes of Figure 6 are $-0.0526,-0.0527$; that is to say, system is stable.

In (40), the changes of steps with $k$ can be seen in Figure 7.

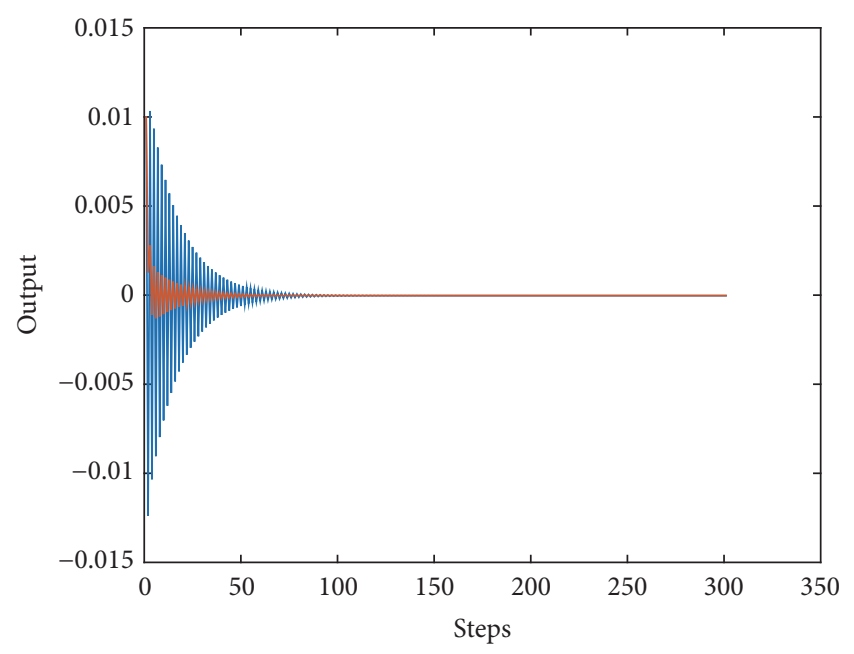

Figure 6: Output with $\varepsilon=1.1$.

It can be seen from Figure 7 that the output of the system has obvious irregular variation for the passive system with optimal function control item, and the output of the system becomes more complicated with the increase of the control coefficient. At this point the fixed point becomes unstable fixed point again, and the system presents chaos. The results are also demonstrated by the following Lyapunov indexes.

Under the selected parameters above, the corresponding Lyapunov indexes of control system are as follows:

(1) $k=0.3$, Lyapunov indexes are 0.5499 and -0.6553 ;

(2) $k=0.35$, Lyapunov indexes are 0.5343 and -0.6396 ;

(3) $k=0.4$, Lyapunov indexes are 0.3188 and -0.4242 ;

(4) $k=0.45$, Lyapunov indexes are 0.5348 and -0.6402 .

The change of Lyapunov indexes of the anticontrol system with $k$ is shown in Figure 8.

It can be shown in Figure 8 that with the anticontrol item, the Lyapunov exponent of $x$ is always positive, indicating that the system is chaotic.

Finally we give the bifurcation figure with the change of the parameter $k$. It can be seen from the Figure 9 that when the anticontrol parameter $k$ is taken as 0.15 , the system output bifurcation occurs. When $k$ is 0.42 , the system output shows obvious chaos. The original stability system becomes a chaotic system with the change of parameter $k$.

\section{Discussion of the Chaotic Game}

For the output duopoly competing evolution model, we consider

$$
z(n+1)=H(z(n))+\mu(z(n))+v(z(n)),
$$




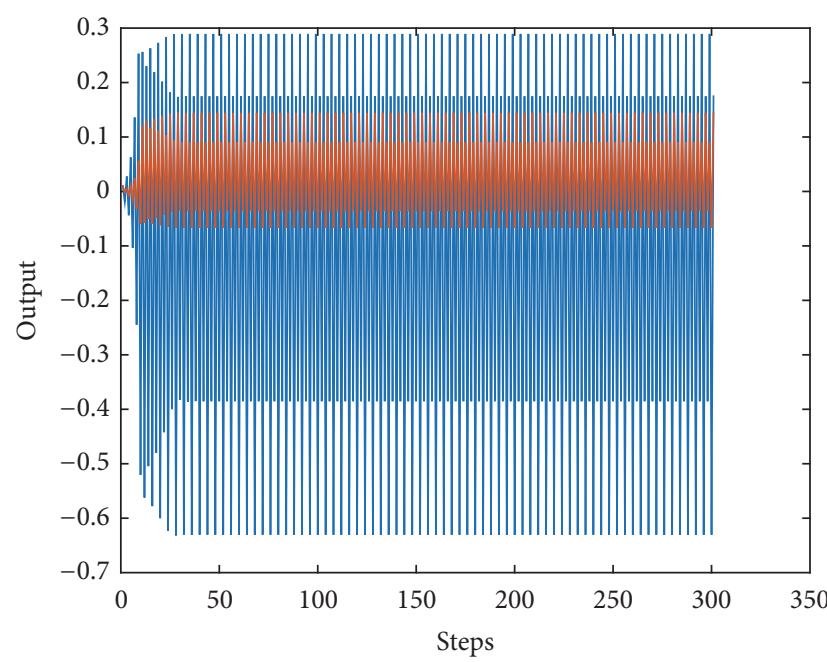

(a)

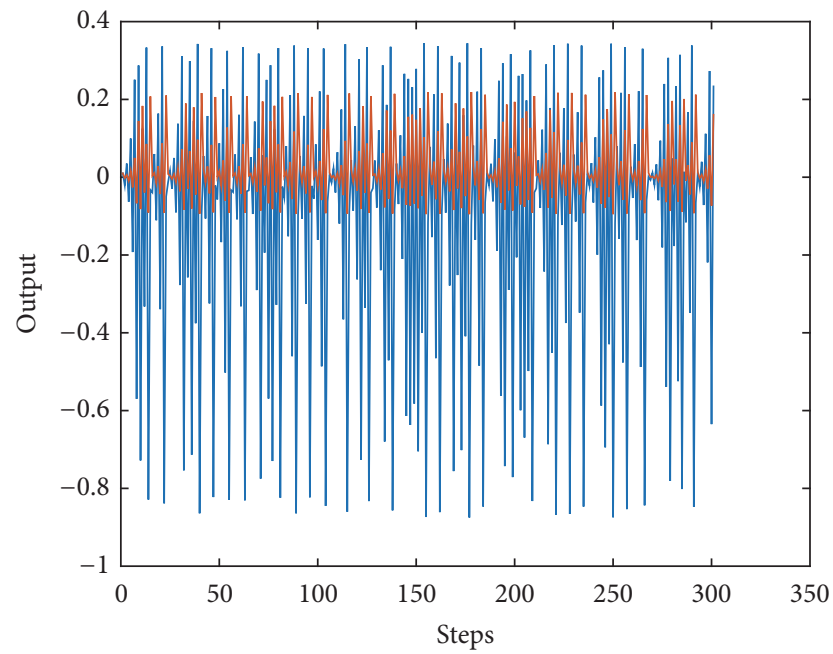

(c)

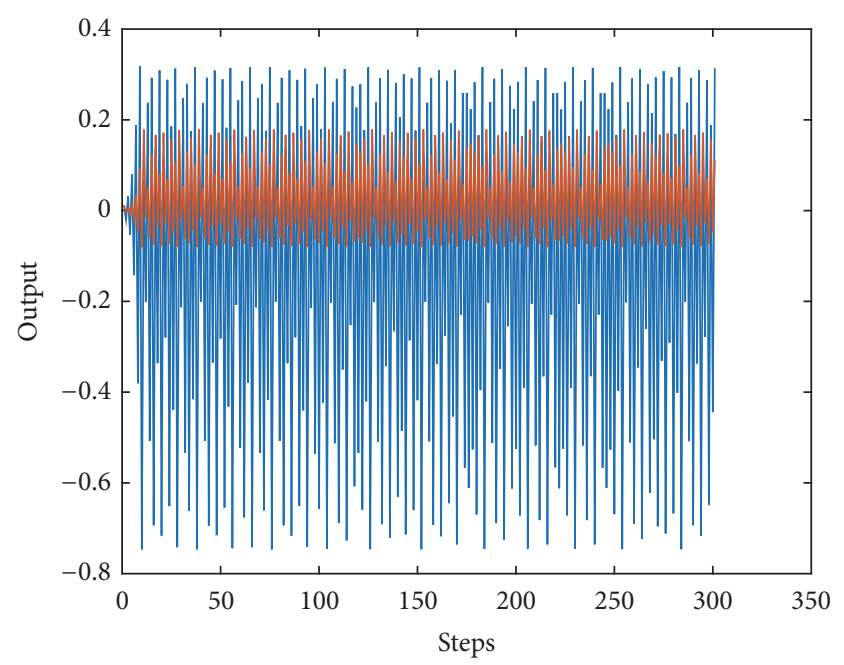

(b)

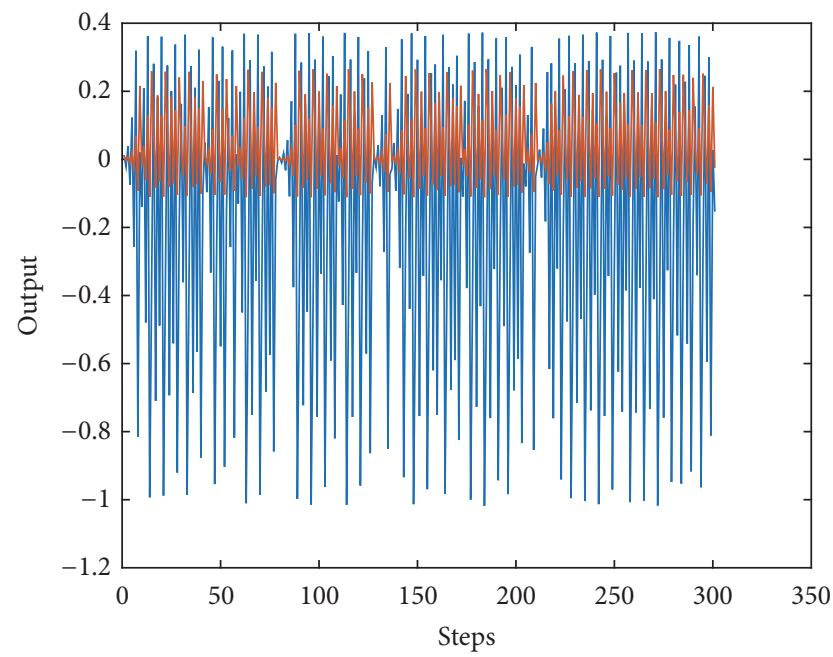

(d)

FIGURE 7: The changes of the output with antichaos control item (a) $k=0.3$; (b) $k=0.35$; (c) $k=0.4$; (d) $k=0.45$.

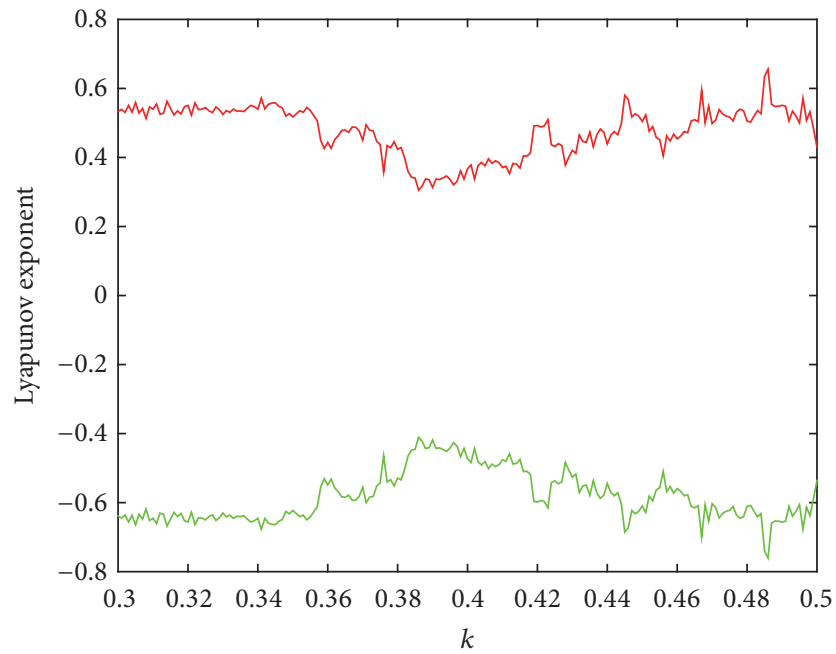

FIgURE 8: The change of Lyapunov exponents with $k$.

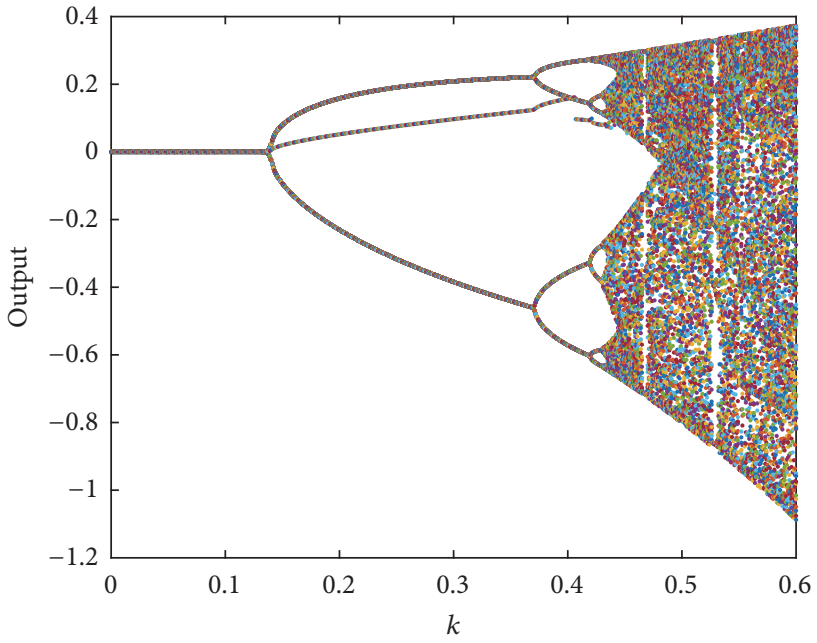

FIGURE 9: The bifurcation of the output with the change of anticontrol parameter $k$. 
where $z(n)=\left(\begin{array}{l}x_{1}(n) \\ x_{2}(n) \\ x_{3}(n)\end{array}\right)$, and $H(z(n))$ is obtained since an-

tichaos control is conducted on system (26), and

$$
H(z(n))=\left(\begin{array}{c}
x_{1}(n)+F\left(x_{1}(n), x_{2}(n), x_{3}(n)\right)+k\left(x_{1}(n)+F\left(x_{1}(n), x_{2}(n), x_{3}(n)\right)-x_{1}(n)\right) \\
x_{2}(n)+G\left(x_{1}(n), x_{2}(n), x_{3}(n)\right)+k\left(x_{2}(n)+G\left(x_{1}(n), x_{2}(n), x_{3}(n)\right)-x_{2}(n)\right) \\
x_{3}(n)-\frac{k \varepsilon}{2}\left[\left(a_{11} V_{31}+a_{21} V_{32}\right) x_{1}(n)+\left(a_{12} V_{31}+a_{22} V_{32}\right) x_{2}(n)+V_{33} x_{3}(n)\right]
\end{array}\right)
$$

$F, G$ have been given in the sixth part and $a_{11}, a_{12}, a_{21}$, and $a_{22}$ are in the fourth part. $V_{11}, V_{12}, V_{21}$, and $V_{22}$ are the corresponding elements of $V . \mu(z(n))$ is for stability and $v(z(n))$ is for chaos.

According to basic thought, there is the following process. When $n=1$,

$$
z(2)=H(z(1))+\mu_{1}+v_{1},
$$

without control items $\mu_{1}$ and $v_{1}$, we have $z(2)=H(z(1))$, which can be rewritten as the standard form similar to (4); that is,

$$
E_{1} z(2)=A_{1} z(1)+l(z(1))
$$

where

$$
\begin{aligned}
& E_{1}=\left(\begin{array}{lll}
1 & 0 & 0 \\
0 & 1 & 0 \\
0 & 0 & 0
\end{array}\right), \\
& A_{1}=\left(\begin{array}{ccc}
1+(k+1)\left[a_{11}-\frac{\varepsilon}{2}\left(a_{11} V_{11}+a_{21} V_{12}\right)\right] & (k+1)\left[a_{1} 2-\frac{\varepsilon}{2}\left(a_{12} V_{11}+a_{22} V_{12}\right)\right] & -\frac{\varepsilon}{2}(k+1) V_{13} \\
(k+1)\left[a_{21}-\frac{\varepsilon}{2}\left(a_{11} V_{21}+a_{21} V_{22}\right)\right] & 1+(k+1)\left[a_{22}-\frac{\varepsilon}{2}\left(a_{12} V_{21}+a_{22} V_{22}\right)\right] & -\frac{\varepsilon}{2}(k+1) V_{23} \\
-\frac{\varepsilon(k+1)}{2}\left(a_{11} V_{31}+a_{21} V_{32}\right) & -\frac{\varepsilon(k+1)}{2}\left(a_{12} V_{31}+a_{22} V_{32}\right) & 1-\frac{\varepsilon(k+1)}{2} V_{33}
\end{array}\right),
\end{aligned}
$$

$l(z(1))=f(x(1))$, and $f(x(k))$ has been given in the second part.

Then the methods above are used to study this problem. When $E_{1} z(2)=A_{1} z(1)+l(z(1))+\mu_{1}$ satisfies the conditions of Theorem 5, there is a state feedback passive controller $\mu_{1}=-\left(\varepsilon_{1} / 2\right) V_{1} A_{1} z(1)$, making $z(2)=H(z(1))+\mu_{1}$ stable, where $\varepsilon_{1}$ is a constant bigger than zero and $V_{1}$ is the only positive semidefinite solution satisfying $A_{1}^{T} V_{1} A_{1}-E_{1}^{T} V_{1} E_{1}=$ $-E_{1}^{T} W E_{1}$.

Then we consider $v_{1}$. Using the method of anticontrol, we have where $M\left(x_{1}(1), x_{2}(1), x_{3}(1)\right)=-\left(k_{1} \varepsilon_{1} / 2\right)\left[\left(a_{11}^{\prime} V_{31}^{\prime}+\right.\right.$ $\left.\left.a_{21}^{\prime} V_{32}^{\prime}\right) x_{1}(1)+\left(a_{12}^{\prime} V_{31}^{\prime}+a_{22}^{\prime} V_{32}^{\prime}\right) x_{2}(1)+V_{33}^{\prime} x_{3}(1)\right], a_{11}^{\prime}=1+$ $\left(k_{1}+1\right)\left[a_{1} 1-(\varepsilon / 2)\left(a_{11} V_{11}+a_{21} V_{12}\right)\right], a_{21}^{\prime}=\left(k_{1}+1\right)\left[a_{21}-\right.$ $\left.(\varepsilon / 2)\left(a_{11} V_{21}+a_{21} V_{22}\right)\right], a_{12}^{\prime}=\left(k_{1}+1\right)\left[a_{12}-(\varepsilon / 2)\left(a_{12} V_{11}+\right.\right.$ $\left.\left.a_{22} V_{12}\right)\right], a_{22}^{\prime}=1+\left(k_{1}+1\right)\left[a_{22}-(\varepsilon / 2)\left(a_{12} V_{21}+a_{22} V_{22}\right)\right]$. $V_{31}^{\prime}, V_{32}^{\prime}$, and $V_{33}^{\prime}$ are corresponding elements of $V_{1}$, because $v_{1}$ is related to $z(1)$ and $M\left(x_{1}(1), x_{2}(1), x_{3}(1)\right)$. And $M\left(x_{1}(1), x_{2}(1), x_{3}(1)\right)$ is related to $\mu_{1}$, so $v_{1}$ is the anticontrol item related to $\mu_{1}$. That is to say, $\mu_{1}$ and $v_{1}$ can enable system (45) to realize the chaos game.

When $n=2, z(3)=H(z(2))+\mu_{2}+v_{2}$. Similar to the above analysis, firstly, we consider $z(3)=H(z(2))$ without control items $\mu_{2}, v_{2}$, which can also be rewritten as the standard 
form like $E_{1} z(2)=A_{1} z(1)+l(z(1))$; then we can get the passive control item like $\mu_{2}=-\left(\varepsilon_{2} / 2\right) V_{2} A_{2} z(2)$. Because of $z(2)=H(z(1))+\mu_{1}+v_{1}$, the choose of $\mu_{2}$ is related to $\mu_{1}, v_{1}$. According to the analysis of $n=1$, we can get the anticontrol item like $v_{1}$, and $v_{2}$ is connected with $z(2)$ and $\mu_{2}$. Thus $v_{2}$ is related to $\mu_{1}, v_{1}$, and $\mu_{2}$.

By similar analysis for the $n$th step, for $z(n+1)=$ $H(z(n))+\mu_{n}+v_{n}$, we can get the passive control item like $\mu_{n}=-\left(\varepsilon_{n} / 2\right) V_{n} A_{n} z(n)$. And according to the above analysis, $\mu_{n}$ is related to $\mu_{1}, v_{1}, \mu_{2}, v_{2}, \ldots, \mu_{n-1}, v_{n-1}$. Furthermore, we get the anticontrol item $v_{n}$ related to $\mu_{1}, v_{1}, \mu_{2}, v_{2}, \ldots, \mu_{n-1}$, $v_{n-1}, \mu_{n}$.

\section{Conclusion}

With the rapid development of social economy, science, and technology, and the evolution of the complex economic system, the traditional theory of economics based on linear system theory is faced with great challenge. Chaos theory mainly studies the influence of "instability," "structural change," on the system, and the causes of "abnormal behavior," which contrasts with the "stable" and "reasonable behavior" that traditional economic theory emphasizes. The traditional economy advocates certainty and balance, believing that the economic system will remain in a quiescent state without external factors and emphasizes the linear response. And chaotic economists proceed with the "imperfect and unbalanced world" to explain the economic system, which is full of fuzziness, chaos, and contingency, and try to find a dialectical relationship of the transition between order and disorder [26]. Therefore, chaos theory opens up a new way for the study of economic chaos behavior, and, with chaos theory, it may reveal the complex dynamic characteristics that can not be explained by traditional methods [2, 27-30]. As a new hot-spot and the highlight in chaos research and application, chaos control has received extensive attention, and it has also been applied to the study of chaos in economics. Competitions, whether in the military or economic markets, are real and always exist. How to use low investment to realize the maximal interests has been the basis that provides a chance for practical uses of game thoughts.

The chaos game of the output duopoly competing evolution model under the given parameters is discussed in this paper. Firstly, we use the passive theory to carry out chaos control. Then, we have chaos anticontrol for the stable system, and the results can be shown by the Lyapunov exponents. And finally, the chaos game about the system is introduced.

\section{Conflicts of Interest}

The authors declare that they have no conflicts of interest.

\section{Acknowledgments}

The research was supported by the National Natural Science Foundation of China (Grant nos. 61403231, 11501328, and 61533011) and China Postdoctoral Science Foundation (Grant no. 2016M592188).

\section{References}

[1] M. J. Stutzer, "Chaotic dynamics and bifurcation in a macro model," Journal of Economic Dynamics \& Control, vol. 2, no. 4, pp. 353-376, 1980.

[2] R. S. Huang and H. Huang, Chaos and its Applications, Wuhan University Press, Hubei, China, 2nd edition, 2005.

[3] F. Wu and J. Ma, "The chaos dynamic of multiproduct Cournot duopoly game with managerial delegation," Discrete Dynamics in Nature and Society, vol. 2014, Article ID 206961, 10 pages, 2014.

[4] M. Akhmet, Z. Akhmetova, and M. O. Fen, "Chaos in economic models with exogenous shocks," Journal of Economic Behavior \& Organization, vol. 106, pp. 95-108, 2014.

[5] E. Ott, C. Grebogi, and J. A. Yorke, "Controlling chaos," Physical Review Letters, vol. 64, no. 11, pp. 1196-1199, 1990.

[6] L. Kass, "Stabilizing chaos in a dynamica macroeconomic model," Journal of Economic Behavior \& Organization, vol. 33, no. 3, pp. 313-332, 1998.

[7] J. Liu, S. Liu, and W. Li, "Complex modified generalized projective synchronization of fractional-order complex chaos and real chaos," Advances in Difference Equations, 2015:274, 16 pages, 2015.

[8] X. J. Zhu and M. D. Liu, "On the oligopoly model and price competition in china's insurance market," Journal of Finance, vol. 3, pp. 80-85, 2003.

[9] S. S. Askar, "Complex dynamic properties of Cournot duopoly games with convex and log-concave demand function," Operations Research Letters, vol. 42, no. 1, pp. 85-90, 2014.

[10] T. Puu, "Complex dynamics with three oligopolists," Chaos, Solitons \& Fractals, vol. 7, no. 12, pp. 2075-2081, 1996.

[11] T. Puu, "On the stability of Cournot equilibrium when the number of competitors increases," Journal of Economic Behavior ఓ Organization, vol. 66, no. 3-4, pp. 445-456, 2008.

[12] G. I. Bischi and A. Naimzada, "Global analysis of a dynamic duopoly game with bounded rationality," in Advances in Dynamic Games and Application, vol. 5, pp. 361-385, Birkhäuser, Basel, Switzerland, 2000.

[13] H. N. Agiza, A. S. Hegazi, and A. A. Elsadany, "The dynamics of Bowley's model with bounded rationality," Chaos, Solitons \& Fractals, vol. 12, no. 9, pp. 1705-1717, 2001.

[14] J.-g. Du, T. Huang, and Z. Sheng, "Analysis of decision-making in economic chaos control," Nonlinear Analysis: Real World Applications, vol. 10, no. 4, pp. 2493-2501, 2009.

[15] J. Du, T. Huang, Z. Sheng et al., "A new method to control chaos in an economic system," Applied Mathematics \& Computation, vol. 217, no. 6, pp. 2370-2380, 2010.

[16] J. Q. Fang, "Control and synchronism of superchaos and chaos," Science \& Technology Review, vol. 14, no. 4, pp. 6-8, 1996.

[17] K. E. Boulding and H. Jarrett, Environmental Quality in a Crowing Economy, Johns Hopkins Press, Baltimore, MD, USA, 1996.

[18] J. Lichtenberg, "Controlling chaos using nonlinear feedback with delay," Physical Review E Statistical Physics Plasmas Fluids \& Related Interdisciplinary Topics, vol. 54, no. 2, pp. 1200-1207, 1996.

[19] J. Du, Y. Fan, Z. Sheng, and Y. Hou, "Dynamics analysis and chaos control of a duopoly game with heterogeneous players and output limiter," Economic Modelling, vol. 33, pp. 507-516, 2013. 
[20] A. A. Elsadany and A. M. Awad, "Dynamical analysis and chaos control in a heterogeneous Kopel duopoly game," Indian Journal of Pure and Applied Mathematics, vol. 47, no. 4, pp. 617-639, 2016.

[21] Y. P. Zhang, Applications and Control of Fractals, Shandong University, Shandong, China, 2008.

[22] N. Yi and H. J. Chen, "Passive control for a class of nonlinear singular discrete systems," Journal of Liaoning Shihua University, vol. 31, no. 3, pp. 74-77, 2011.

[23] M. Yu, Selected Studies on Petroleum Economics, Petroleum Industry Press, Beijing, China, 1999.

[24] H. G. Zhang, Z. L. Wang, and W. Huang, The Control Theory of Chaotic Systems, Northeastern University Press, Liaoning, China, 2003.

[25] B. Men, X. S. Li, and Q. L. Zhang, "Permitted control condition for discrete singular systems," Journal of Shenyang Normal University (Natural Science Edition), vol. 24, no. 2, pp. 132-135, 2006.

[26] Z. H. Shi, "Comparative study of traditional economics and chaos economics - and on the limitations of traditional economic theory," Foreign Economies \& Management, vol. 23, no. 10, pp. 13-16, 2001.

[27] G. Hu, Chaos Control, Shanghai Scientific and Technical Education Press, Shanghai, China, 2000.

[28] X. S. Luo, Chaos Synchronization, Control And Its Application, Guangxi Normal University Press, Guangxi, China, 2007.

[29] Z. Wang, X. Huang, and G. D. Shi, "Analysis of nonlinear dynamics and chaos in a fractional order financial system with time delay," Computers \& Mathematics with Applications, vol. 62, no. 3, pp. 1531-1539, 2011.

[30] Y. Li, X. Huang, Y. Song, and J. Lin, "A new fourth-order memristive chaotic system and its generation," International Journal of Bifurcation and Chaos, vol. 25, no. 11, Article ID 1550151, 1550151, 9 pages, 2015. 


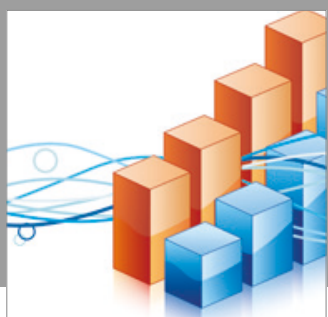

Advances in

Operations Research

vatersals

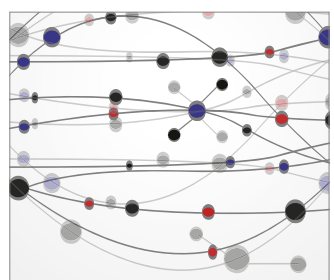

\section{The Scientific} World Journal
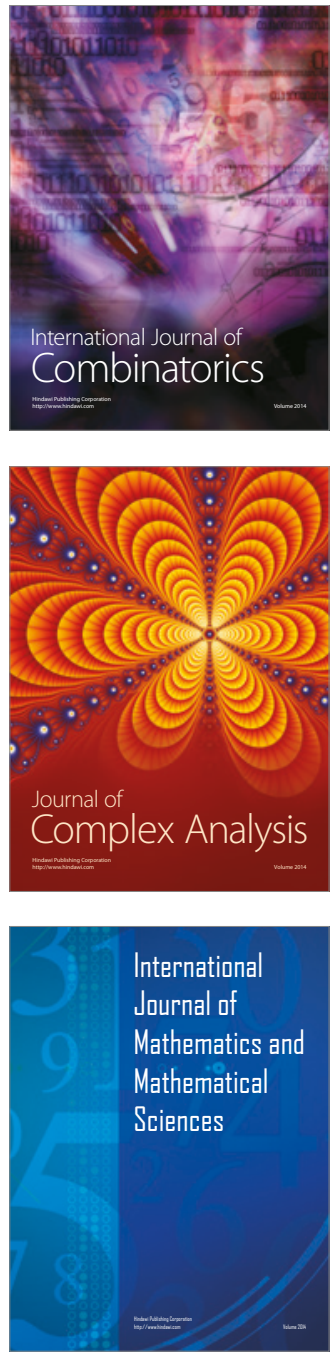
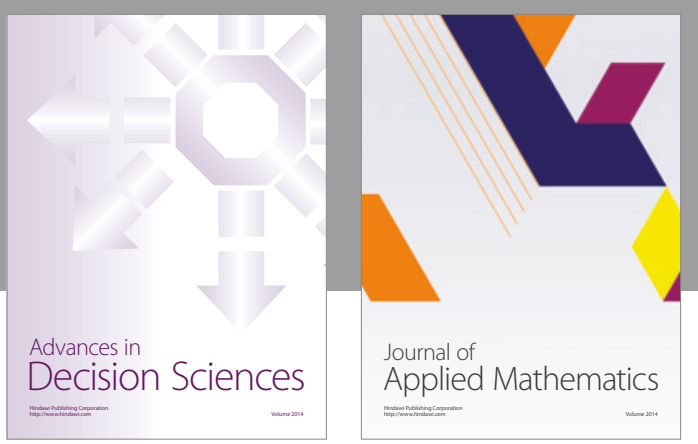

Algebra

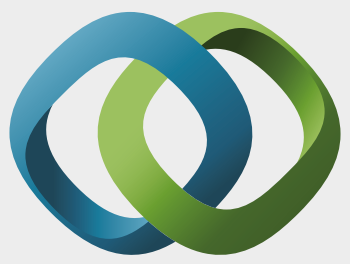

\section{Hindawi}

Submit your manuscripts at

https://www.hindawi.com
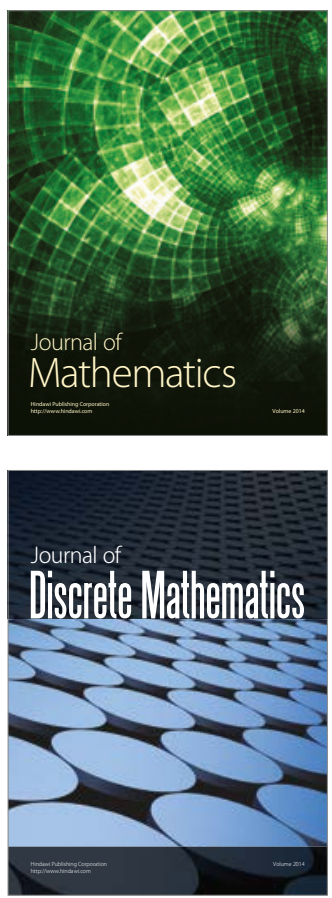

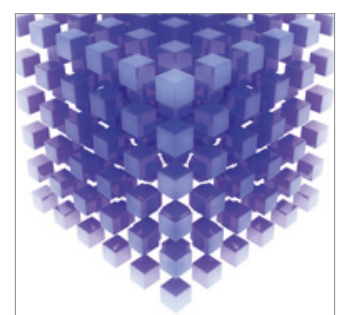

Mathematical Problems in Engineering
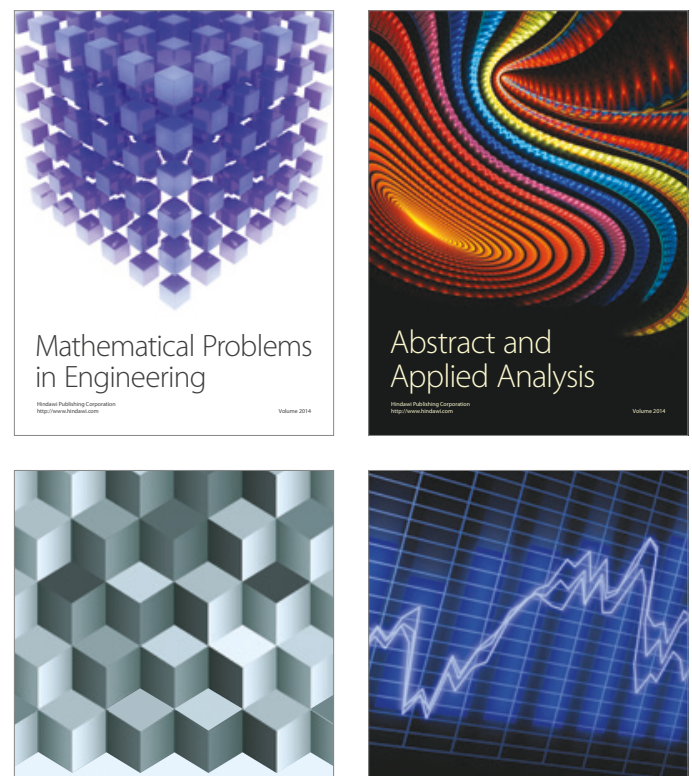

Journal of

Function Spaces

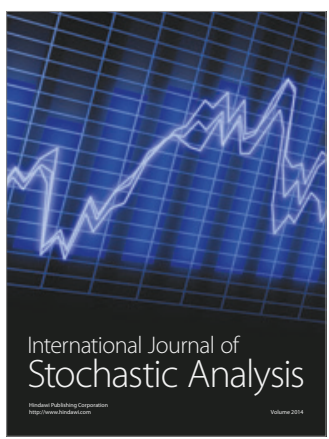

Probability and Statistics
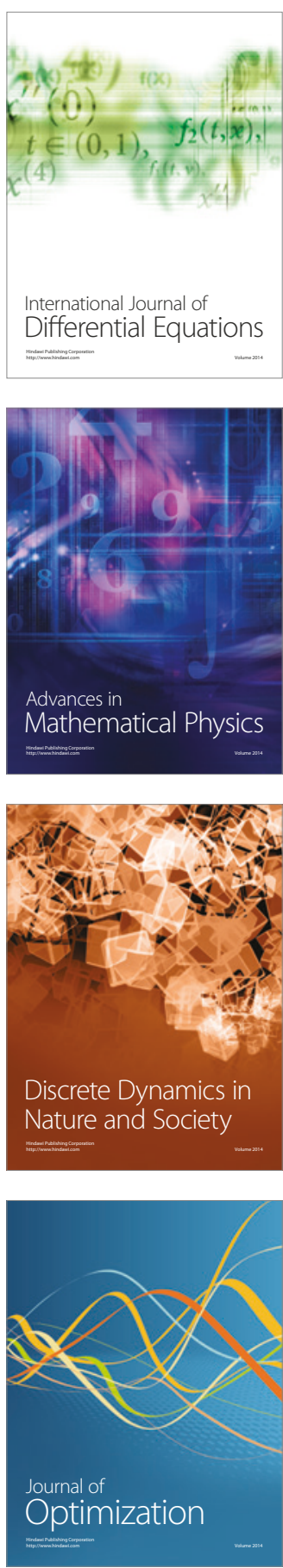